DOI:

10.1038/nrm2404

\title{
Technology watch
}

\section{INTRODUCING BAC TRANSGENEOMICS}

Poser et al. describe an efficient, generic and scalable approach to study protein function in mammalian cells based on bacterial artificial chromosome (BAC)-based transgenesis.

Protein tagging in mammalian culture cells is typically done with cDNA-based transgenes that lack the normal non-coding regulatory information and that are usually driven by unrelated ubiquitous or tissue-specific promoters. By contrast, the large size of the BAC transgene ensures the presence of most regulatory elements and results in expression that closely matches that of the endogenous gene. Poser et al. showed that BAC transgenes can be rapidly and reliably generated using a 96-well format, and established protocols for efficient, stable transfection of these large constructs. Next, the authors selected 15 well-characterized genes and reproduced the known localization patterns for 11 of them. The analysis highlighted the importance of tagging proteins at both termini. BAC transgenes can also be used to study protein-protein and/or protein-DNA interactions as well as protein function during development and in adult organisms. Perhaps the most important step forward with this approach is that it can be easily transferred to any model system that allows stable transgenesis.

ORIGINAL RESEARCH PAPER Poser, I. et al. BAC TransgeneOmics: a high throughput method for exploration of protein function in mammals. Nature Meth. 6 April 2008 (doi:10.1038/NMETH.1199)

\section{NONINVASIVE LIVE IMAGING}

Molecular imaging technologies that use bioluminescence and fluorescence strategies continue to evolve rapidly. However, no single technique currently meets the needs of high sensitivity, high spatial and temporal resolution and high throughput. In an attempt to solve some of the limitations of fluorescence imaging in living subjects, Keren et al. report a strategy for small-animal optical imaging based on Raman spectroscopy.

Raman spectroscopy can differentiate the spectral fingerprint of many molecules, resulting in very high multiplexing capabilities. Narrow spectral features are easily separated from the broadband autofluorescence, because Raman is a scattering rather than an absorption/emission phenomenon, and Raman active molecules are more photostable than fluorophores, which are rapidly photobleached. In this study, the authors adapted a Raman microscope to demonstrate Raman imaging of small living subjects while using two different types of Raman nanoparticles - surface enhanced Raman scattering (SERS) active nanoparticles and single-walled carbon nanotube (SWNT) nanoparticles - both of which result in high Raman intensities. This method was used successfully to study pharmacokinetics of nanoparticles, in vivo tumour targeting after tail-vein injection and whole-body deep-tissue imaging. This imaging method holds significant potential as a strategy for biomedical imaging of living subjects.

ORIGINAL RESEARCH PAPER Keren, S. et al. Noninvasive molecula imaging of small living subjects using Raman spectroscopy. Proc. Natl Acad. Sci. USA 31 March 2008 (doi:10.1073/pnas.0710575105) 